\title{
Leakage of an Invagination Pancreaticojejunostomy May Have an Influence on Mortality
}

\author{
Harish Lavu, ${ }^{1, *}$ Neal McCall, ${ }^{1}$ Scott W. Keith, ${ }^{2}$ Elizabeth M. Kilbane, ${ }^{3}$ Abhishek D. Parmar, ${ }^{4}$ Bruce L. Hall, ${ }^{5}$ and Henry A. Pitt ${ }^{6}$
}

\begin{abstract}
Purpose: No consensus exists regarding the most effective form of pancreaticojejunostomy (PJ) following pancreaticoduodenectomy (PD).

Methods: Data were gathered through the American College of Surgeons-National Surgical Quality Improvement Program, Pancreatectomy Demonstration Project. A total of 1781 patients underwent a PD at 43 institutions. After appropriate exclusions, 890 patients were analyzed. Patients were divided into duct-to-mucosa ( $n=734,82 \%)$ and invagination $(n=156,18 \%)$ groups and were compared by unadjusted analysis. Type of PJ was included in eight separate morbidity and mortality multivariable analyses.

Results: Invagination patients had higher serum albumin $(p<0.01)$ and lower body mass index $(p<0.01)$, were less likely to have a preoperative biliary stent $(p<0.01)$, and were more likely to have a soft gland $(p<0.01)$. PJ anastomosis type was not associated with morbidity but was associated with mortality (duct-to-mucosa vs. invagination, odds ratio $=0.22, p<0.01$ ). Among patients who developed a clinically relevant pancreatic fistula, none of the 119 duct-to-mucosa, compared with 5 of 21 invagination, patients died $(p<0.01)$.

Conclusion: Patients who undergo a PJ by duct-to-mucosa or invagination differ with respect to preoperative and intraoperative variables. When an invagination PJ leaks, there may be a greater influence on mortality than when a duct-to-mucosa PJ leaks.
\end{abstract}

Keywords: pancreatic adenocarcinoma, pancreatic fistula, pancreaticoduodenectomy, pancreaticojejunostomy

\section{Introduction}

Patients with pancreatic ductal adenocarcinoma (PDA) have an $8 \%$ overall 5 -year survival rate, with the most effective current therapy being primary tumor resection. ${ }^{1,2}$ Pancreaticoduodenectomy (PD) is the most common surgical procedure performed for resection of PDA; however, this complex operation is associated with a high perioperative complication rate. The most common significant complication associated with PD is a postoperative pancreatic fistula (POPF), which is a leakage of amylase-rich fluid from the site of the pancreaticojejunostomy (PJ). ${ }^{1,3-5}$ Among the three anastomoses performed for pancreatic reconstruction, the $\mathrm{PJ}$ is generally considered the "Achilles' heel" of PD, due not only to the relatively high incidence of leakage but also the significance in terms of patient recovery. ${ }^{6}$ Research studies and reviews generally place the incidence of clinically significant POPF to be between $10 \%$ and $30 \%$. Although not commonly seen, very severe uncontrolled POPF can even lead to postoperative mortality. ${ }^{7,8}$ Studies have reported mortality rates related to severe POPF ranging from $20 \%$ to $40 \% .{ }^{4,9,10}$ Given this high risk, an emphasis on decreasing complications associated with the PJ anastomosis is needed to maximize the curative benefit of PD.

\footnotetext{
Departments of ${ }^{1}$ Surgery and ${ }^{2}$ Biostatistics, Thomas Jefferson University, Philadelphia, Pennsylvania.

${ }^{3}$ Indiana University Hospital, IU Health, Surgery, Indianapolis, Indiana.

${ }^{4}$ University of Texas Medical Branch, Surgery, Galveston, Texas.

${ }^{5}$ Washington University, School of Medicine, St. Louis, Missouri.

${ }^{6}$ Lewis Katz School of Medicine at Temple University, Surgery, Philadelphia, Pennsylvania.

Presented at 14th annual meeting of the AHPBA, March 2015, Miami, FL, and 45th annual meeting of the Pancreas Club, May 2015, Washington, DC.

*Address correspondence to: Harish Lavu, MD, Department of Surgery, Thomas Jefferson University, 1025 Walnut Street, Suite 605, Philadelphia, PA 19107, E-mail: harish.lavu@jefferson.edu
}

(C) Harish Lavu et al. 2018; Published by Mary Ann Liebert, Inc. This Open Access article is distributed under the terms of the Creative Commons License (http://creativecommons.org/licenses/by/4.0), which permits unrestricted use, distribution, and reproduction in any medium, provided the original work is properly cited. 
Considerable efforts have been made to reduce the incidence of PF in the past 30 years. Fistula mitigation strategies include alternative anastomosis techniques, the use and management of intraperitoneal drains, fibrin glue, and pharmacological agents, among others. None of these methods has proven to be definitively effective. ${ }^{11-14}$ The two standard techniques to restore gastrointestinal continuity for the pancreas are the invagination PJ (IPJ), also known as the "dunking technique," and the duct-to-mucosa pancreaticojejunostomy (DmPJ). In the IPJ method, the surgeon opens a portion of the jejunum sufficient to "dunk" the pancreatic stump into the side of the jejunum. This technique requires a larger jejunotomy than the competing DmPJ method, where the surgeon makes a small jejunotomy that corresponds only to the size of the pancreatic duct. Sutures are placed directly between the pancreatic duct and the jejunal mucosa, allowing for close adhesion of the two layers. ${ }^{15-17}$ A number of studies have compared the effectiveness of DmPJ and IPJ. One retrospective, single-institution study revealed a $3.2 \%$ rate of POPF in the DmPJ group and a rate of $17.5 \%$ in its IPJ group without significant mortality differences. $^{18}$ One dual-institution controlled trial, however, found an odds ratio (OR) of 2.4 for POPF incidence between DmPJ and IPJ, again without significant mortality differences. ${ }^{6}$ Still, other studies have reported minimal to no difference in risk of fistula formation or mortality. ${ }^{15,19,20}$ One of the limitations to prior studies is that they were restricted to single or small multi-institutional trials. Given the contradicting conclusions of previous research, more investigation into the effectiveness of these techniques for pancreatic anastomotic reconstruction is warranted.

In this study, we aim to retrospectively compare morbidity and mortality between invagination and duct-to-mucosa PJ from the multi-institutional American College of Surgeons-National Surgical Quality Improvement Program (ACS-NSQIP) Pancreatectomy Demonstration Project (PDP).

\section{Methods}

Pancreatectomy demonstration project

A multicenter, retrospective cohort study was performed to evaluate morbidity and mortality between invagination and duct-to-mucosa pancreaticojejunostomy. This retrospective analysis was considered exempt by the Institutional Review Board of Thomas Jefferson University. Data were gathered through the ACS-NSQIP, PDP. This initiative gathered pancreatectomy-specific data from 43 participating hospitals (see the Acknowl- edgments section). Data were collected from November 1, 2011, through December 31, 2012. Data collection was standardized by utilizing trained surgical clinical reviewers (SCRs) within ACS-NSQIP. The SCRs have ongoing data audits and follow precise definitions for data characteristics. Procedures are recorded into the ACS-NSQIP database using Current Procedural Technology codes. These processes have been proven to ensure effective, high-volume data entry into the NSQIP database. ${ }^{21}$ Further details regarding the PDP have previously been published. ${ }^{2-26}$

\section{Study patients}

During the study period, 2805 patients underwent a pancreatic resection at the 43 participating institutions. Patients who underwent total pancreatectomy, distal pancreatectomy, enucleation, and minimally invasive PD were excluded. Patients who lacked data on gland texture and duct size also were excluded, given the significance of these two factors in POPF formation. ${ }^{27,28}$ As a result, 890 patients were available for analysis. Data on patient demographics and comorbidities, preoperative laboratory values, intraoperative characteristics, and postoperative outcomes were collected. Definitions and instructions for data collection of these variables can be found in the ACS-NSQIP Data Users Guide. ${ }^{29}$ Patients were divided into DmPJ and IPJ groups and were compared by unadjusted univariate analyses. Type of PJ was then included in eight separate morbidity and mortality multivariable analyses.

\section{Outcome measures}

All outcomes recorded in the ACS-NSQIP were assessed 30 days postoperatively, except mortality, which could be indicated at any time postdischarge. The primary outcomes of this study were perioperative overall morbidity, serious morbidity as previously defined, ${ }^{22-26}$ mortality, and POPF-associated mortality. Aside from the variables collected by general participation in ACS-NSQIP, the 43 institutions participating in the PDP recorded 24 additional pancreatectomyspecific variables. ${ }^{22-25}$ The PDP-specific variables included in this study were preoperative biliary stenting, chemotherapy and radiation 90 days before PD, pancreatic texture, pancreatic duct size, vascular resection, pancreatic reconstruction method (DmPJ or IPJ), intraperitoneal drain placement, drain removal, drain amylase, delayed gastric emptying, POPF, percutaneous drainage, and presence of malignant histology. ACS-NSQIP PDP definitions have been published in the ACS-NSQIP Procedure Targeted Pancreatectomy 
Variables and Definitions. ${ }^{29}$ POPF was defined by two scenarios: (1) persistent drain output of amylase-rich fluid of three times the concentration of serum amylase on or after POD 3 in addition to one of the following (drain continuation for longer than 7 days, percutaneous drainage was performed, or reoperation was required) or (2) the clinical diagnosis of POPF by the attending surgeon as well as drain continuation for longer than 7 days, presence of spontaneous wound drainage, percutaneous drainage was performed, or reoperation was required.

\section{Data analysis}

Continuous study variables were summarized between reconstruction groups by their medians and the first and third quartiles and tested for significant difference by Wilcoxon rank sum tests. Categorical study variables were summarized between reconstruction groups by their frequencies and percentages and tested for significant dependencies by Fisher's exact tests. Logistic regression models were used to evaluate the likelihood of binary endpoints (overall mortality during followup, overall morbidity, pancreatic fistula, and reoperation) associated with reconstruction (i.e., DmPJ vs. IPJ). Covariates included age (categorized in 10-year groups), body mass index (BMI: $\mathrm{kg} / \mathrm{m}^{2}$ categorized using World Health Organization guidelines for underweight, normal weight, overweight, obese I, and obese II), sex, preoperative biliary stent, albumin (below vs. $\geq 3.5 \mathrm{~g} / \mathrm{dL}$ ), texture (soft, intermediate, or hard), and duct size (under 3, 3-6, and $>6 \mathrm{~mm}$ ). Due to the small number of mortality events observed, we used a stepwise selection procedure to identify a model with 3 (or fewer) parameters. The significance level for all tests was 0.05 , and all analyses were conducted in SAS version 9.4 (Cary, NC).

\section{Results}

Study population

During the 14-month study period, 1781 patients underwent $\mathrm{PD}$ at 43 participating institutions. After exclusions as outlined above, 890 patients were available for analysis. Of these patients, 735 (82\%) underwent DmPJ and 156 (18\%) underwent IPJ. Age and gender were similar between DmPJ and IPJ groups (Table 1). The DmPJ patients were found to have a significantly lower median serum albumin ( 3.8 vs. $4.1 \mathrm{~g} / \mathrm{dL}, p<0.01$ ), higher median serum bilirubin level $(0.80$ vs. $0.60 \mathrm{~g} / \mathrm{dL}, p=0.02)$, and higher median BMI (26.2 vs. 24.6, $p=0.01$ ). DmPJ patients also were more likely to have a preoperative biliary stent $(53 \%$ vs. $41 \%, p=0.01)$ and were more likely to have
Table 1. Patient Demographics and Preoperative Variables for 890 Patients

\begin{tabular}{|c|c|c|c|}
\hline & Duct-to-mucosal & Invagination & \\
\hline & $n=734(82 \%)$ & $n=156(18 \%)$ & $p$ \\
\hline Age (years) ${ }^{a}$ & $65.8[57.4,73.1]$ & $65.9[59.6,73.4]$ & 0.50 \\
\hline Male & $398(54 \%)$ & $81(52 \%)$ & 0.66 \\
\hline $\mathrm{BMI}^{\mathrm{a}}$ & $26.2[22.9,30.4]$ & $24.5[22.3,28.6]$ & 0.01 \\
\hline Diabetes mellitus & $196(27 \%)$ & $30(19 \%)$ & 0.10 \\
\hline Cigarette smoking & $163(22 \%)$ & 30 (19\%) & 0.45 \\
\hline Biliary stent & $386(53 \%)$ & $64(41 \%)$ & 0.01 \\
\hline Preoperative chemotherapy & $66(9 \%)$ & $6(4 \%)$ & 0.04 \\
\hline Preoperative radiation & $34(5 \%)$ & $2(1 \%)$ & 0.07 \\
\hline Albumin $(\mathrm{g} / \mathrm{dL})^{\mathrm{a}}$ & $3.8[3.30,4.20]$ & $4.1[3.7,4.4]$ & 0.01 \\
\hline Bilirubin $(\mathrm{g} / \mathrm{dL})^{\mathrm{a}}$ & $0.80[0.50,2.0]$ & $0.60[0.40,1.80]$ & 0.02 \\
\hline BUN (g/dL) ${ }^{a}$ & $13[10,18]$ & $15[11,20]$ & 0.02 \\
\hline
\end{tabular}

${ }^{\text {a }}$ Summarized by median with [first, third quartiles].

BMI, body mass index; BUN, blood urea nitrogen.

undergone neoadjuvant chemotherapy (DmPJ: 9\% vs. $4 \%, p=0.04)$. Intraoperatively, DmPJ patients had different malignant histologies ( $84 \%$ vs. $79 \%$ cancer or intraductal papillary mucinous neoplasm (IPMN), $12 \%$ vs. $8 \%$ neuroendocrine tumor, $p<0.05)$, but were less likely to have a soft gland ( $36 \%$ vs. $47 \%, p<0.01$; Table 2 ).

\section{Outcomes}

Overall morbidity and serious morbidity were similar between the two groups (Table 3; Fig. 1). Patients in each group developed complications, including POPF, at similar rates. Both groups also underwent postoperative percutaneous drainage and reoperation at similar rates. However, type of PJ did not influence any of these morbidity outcomes (Tables 3 and 4). On the contrary, overall mortality was higher in invagination patients ( $1 \%$ vs. $5 \%, p=0.02$, Fig. 2). In a logistic regression model adjusted for selected covariates (including sex and albumin indicator as covariates), DmPJ was associated with about a fifth the odds of mortality compared with IPJ (OR= 0.22 , 95\% confidence interval [0.08-0.64], $p<0.01$; Table 4). Among patients who developed POPF, none

Table 2. Intraoperative Variables

\begin{tabular}{lccc}
\hline & Duct-to-mucosal, $\boldsymbol{n}(\%)$ & Invagination, $\boldsymbol{n}(\%)$ & $\boldsymbol{p}$ \\
\hline Soft gland texture & $261(36)$ & $74(47)$ & 0.01 \\
Duct size <3 mm & $234(32)$ & $53(34)$ & 0.78 \\
Vascular resection & $90(13)$ & $19(13)$ & 1.00 \\
Antecholic DJ/GJ & $413(65)$ & $46(39)$ & 0.01 \\
Drain placement & $577(81)$ & $137(92)$ & 0.01 \\
Clean contaminated & $609(83)$ & $148(95)$ & 0.01 \\
Malignant histology & & & \\
$\quad$ CA or IPMN & $244(84)$ & $38(79)$ & 0.05 \\
$\quad$ Neuroendocrine & $34(12)$ & $4(8)$ & \\
Other & $11(4)$ & $6(13)$ & \\
\hline
\end{tabular}

CA, cancer antigen; DJ/GJ, duodenojejunostomy/gastrojejunostomy; IPMN, intraductal papillary mucinous neoplasm. 
Table 3. Perioperative Outcomes

\begin{tabular}{lccc}
\hline & Duct-to-mucosal, $\boldsymbol{n}$ (\%) & Invagination, $\boldsymbol{n}$ (\%) & $\boldsymbol{p}$ \\
\hline Overall morbidity & $372(51)$ & $68(44)$ & 0.11 \\
Serious morbidity & $323(44)$ & $58(37)$ & 0.13 \\
POPF & $120(17)$ & $21(14)$ & 0.54 \\
Any SSI & $160(22)$ & $29(19)$ & 0.45 \\
Organ space SSI & $67(9)$ & $18(12)$ & 0.37 \\
DGE & $123(17)$ & $24(16)$ & 0.90 \\
DVT & $11(2)$ & $5(3)$ & 0.18 \\
Percutaneous drain & $63(9)$ & $21(15)$ & 0.07 \\
Reoperation $^{\text {a }}$ & $19(3)$ & $6(4)$ & 0.43 \\
Length of stay $^{a}$ & $8[7,13]$ & $8[6,11]$ & 0.13 \\
\hline
\end{tabular}

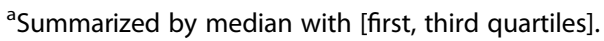

DGE, delayed gastric emptying; DVT, deep vein thrombosis; POPF, postoperative pancreatic fistula; SSI, surgical-site infection.

of the $119 \mathrm{DmPJ}$ patients, compared with 5 of 21 invagination patients, suffered postoperative mortality $(0 \%$ vs. $24 \% p<0.01$; Fig. 2).

\section{Discussion}

POPF is among the most serious complications associated with PD and has broad implications for patient outcomes and recovery from this complex operation. In this multi-institution, retrospective cohort study, we evaluated the influence of the type of PJ on morbidity and mortality. Our analysis suggests an increased risk of overall and POPF-related mortality associated with IPJ compared with DmPJ. These data were adjusted for age, gender, BMI, preoperative albumin levels, and placement of biliary stents, and gland texture and duct diameter via multivariate analysis. The absence of
POPF-associated mortality in the DmPJ group compared with the $24 \%$ POPF-associated mortality in the IPJ group $(p<0.01)$ suggests that DmPJ may be the safer technique of pancreatic reconstruction.

Surgeon training, judgment, and comfort level are important variables that go into the consideration of the type of PJ anastomosis to perform. ${ }^{30-32}$ Some authorities argue that no single method of PJ can be applied ubiquitously to all patients and that tailoring the method of PJ construction to the patient and the type of gland is the best way to decrease POPF. ${ }^{10,28}$ Small duct diameter and soft pancreatic texture are well documented to increase the risk of complication during pancreatic reconstruction. ${ }^{6,7,20,30,33,34}$

The pathological cause of POPF in instances of small duct diameter and soft pancreatic texture may be fundamentally different depending on the method of reconstruction. Small duct diameter is more likely to lead to improper placement of sutures in the DmPJ anastomosis leading to the potential for distraction of flow across the anastomosis resulting in leakage of pancreatic juice. Whereas in the IPJ anastomosis, the extensive suture placement and necessary compression required to perform the invagination technique in patients with soft pancreatic tissue can cause ischemia, laceration, and dehiscence resulting in a POPF. ${ }^{28,30,35}$ The IPJ also juxtaposes the open end of the pancreatic remnant to the intestinal lumen. This architecture increases the likelihood for enzymatic erosion of the

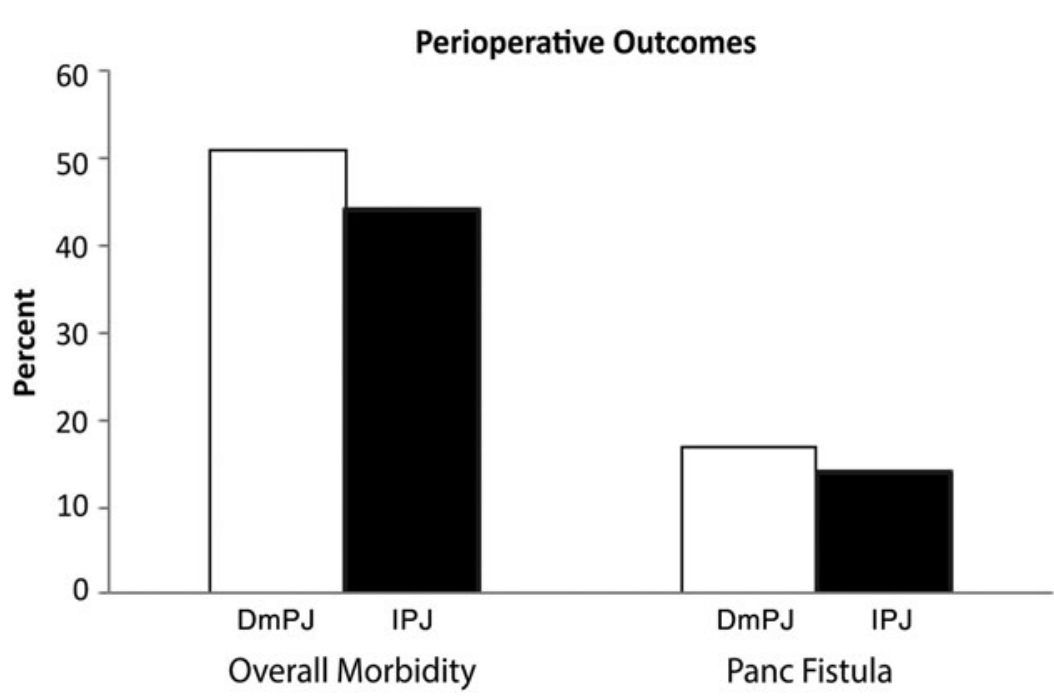

FIG. 1. Postoperative outcomes in patients receiving DmPJ vs. IPJ reconstruction. DmPJ, duct-to-mucosa pancreaticojejunostomy; IPJ, invagination pancreaticojejunostomy. 
Table 4. Logistic Regression Modeling

\begin{tabular}{lcccc}
\hline & \multicolumn{2}{c}{ DmPJ vs. IPJ } & & \\
\cline { 2 - 2 } Outcome variable & OR & & $95 \% \mathrm{Cl}$ & $\boldsymbol{p}$ \\
\hline Overall mortality & 0.22 & & $(0.08-0.64)$ & 0.01 \\
Overall morbidity & 1.22 & & $(0.84-1.76)$ & 0.30 \\
Pancreatic fistula & 1.35 & & $(0.78-2.34)$ & 0.28 \\
Reoperation & 0.66 & & $(0.26-1.68)$ & 0.38 \\
\hline
\end{tabular}

Each row represents an indicator outcome regressed on a proceduretype indicator $(\mathrm{DmPJ}=1, \mathrm{PJ}=0)$ and covariates.

$\mathrm{Cl}$, confidence interval; DmPJ, duct-to-mucosa pancreaticojejunostomy; IPJ, invagination pancreaticojejunostomy; OR, odds ratio.

pancreatic tissue, potentially causing necrosis, stenosis, and subsequently POPF. ${ }^{29,34}$ Given the larger opening in the jejunum associated with an IPJ anastomosis, in the event of leakage, bile intermixed with pancreatic juice may lead to further damage of surrounding tissues. This situation may be more likely to result in an organ space infection, sepsis, septic shock, and/or hemorrhage from the gastroduodenal artery stump or other visceral vasculatures.

Studies comparing DmPJ with IPJ after PD have focused primarily on POPF incidence as opposed to fistularelated mortality. In the study by El Nakeeb et al., ${ }^{28} 107$ patients were randomized into either end-to-side IPJ or DmPJ groups after pancreatic resection. Patients were followed for 1 year postoperatively, and subsequent analysis revealed no significant difference in POPF between DmPJ and IPJ. ${ }^{28}$ Three mortalities were reported in the DmPJ group and four in the IPJ group, with only some attributable to POPF. No subgroup analysis was carried out on the causes of mortality. ${ }^{29}$

In the largest controlled trial of its type, Berger et al. ${ }^{6}$ randomized 197 patients from two institutions into end-to-side IPJ and DmPJ groups. Analysis revealed a $24 \%$ POPF rate in the DmPJ group with a $12 \%$ POPF in the IPJ group, and this difference was statistically significant. To our knowledge, this study is the only one to have demonstrated a higher POPF rate with DmPJ. Mortality was low in this study and comparable between reconstruction methods- $2 \%$ for $\mathrm{DmPJ}$ and $0 \%$ for IPJ. ${ }^{6}$ Both mortalities in the DmPJ group were directly attributable to POPF. In addition, the study also reported significant differences in POPF rates between institutions as well as individual surgeons.

Based on the lack of a consensus in the literature of the superior reconstruction method, we hypothesized that a larger data set, including a diversity of surgeons and institutions, may reveal additional information regarding POPF incidence, overall mortality, and POPF-associated mortality between DmPJ and IPJ. Previous controlled trials were designed to detect POPF incidence differences between techniques as a primary outcome. Consequently, the largest trial had $<100$ patients in each group which, given the already low mortality rates at high-volume centers, limited the assessment of mortality between techniques. Our retrospective analysis is the only study to have demonstrated a significantly increased mortality risk in POPF patients undergoing the IPJ technique.

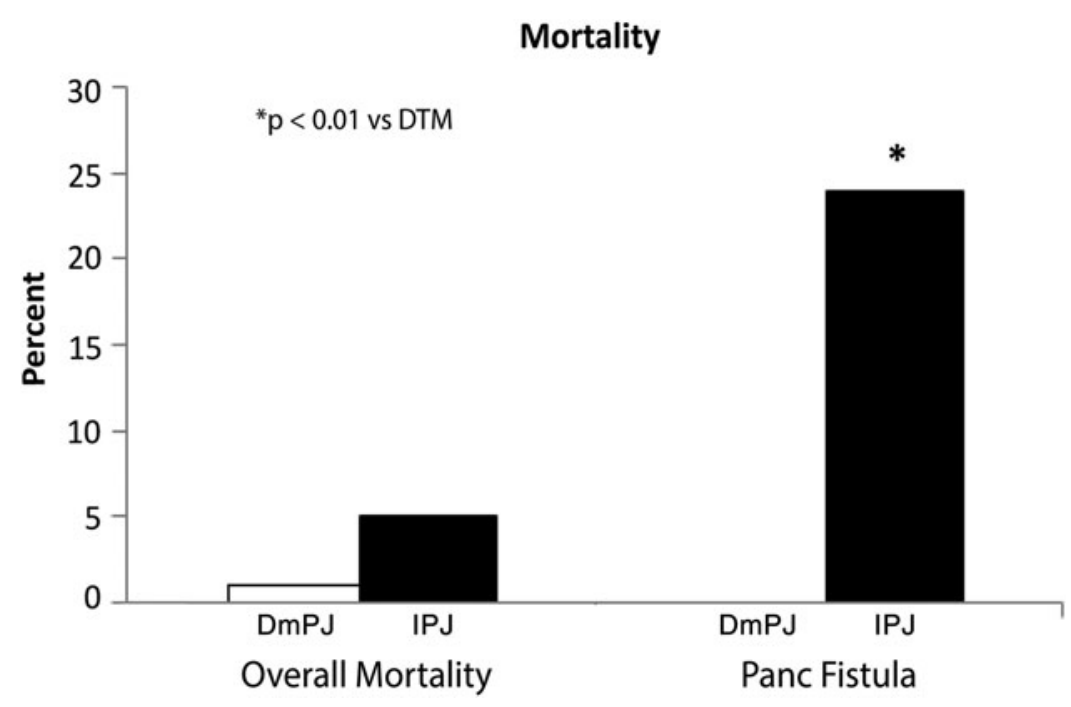

FIG. 2. Overall postoperative mortality (among all patients) and mortality following postoperative pancreatic fistula in patients receiving DmPJ vs. IPJ reconstruction. 
Several limitations are inherent to this retrospective cohort study. First, data were retrieved from many surgeons at many institutions, and studies have reported a wide variation in outcomes based on surgeon skill, experience, volume, and comfort level. ${ }^{6,26}$ However, potential skew in the data was balanced given that the ACS-NSQIP PDP has input from 43 institutions. Second, this nonrandomized retrospective study is subject to selection bias. Whether a particular method of pancreatic reconstruction was selected based on the characteristics of the pancreatic remnant intraoperatively is unknown. For example, surgeons may have reserved one method of reconstruction for patients deemed to be at greater risk of POPF or other complications. The differences in preoperative and intraoperative characteristics may suggest this bias. However, the statistical methodology used in this study controlled for many risk factors, which, in general, would have led to more POPFs in the DmPJ group. The differences between the groups, including the factors of BMI, preoperative biliary stenting, and albumin, would tend to favor the invagination group as it pertains to postoperative complications.

Third, despite multivariate analysis and exclusion of patients without complete data, outcomes measured could have been influenced by confounding factors not recorded in the database. For example, surgeon experience and hospital volume data were not available. Fourth, some parameters measured, such as gland texture, are very difficult to standardize and left to the judgment of the surgeons even with specific ACS-NSQIP definitions. Fifth, with respect to mortality, relatively small numbers are available from which to draw conclusions, and a limitation of the NSQIP database is that we do not have information on the actual cause of death. We can report the association of death with type of anastomosis performed but cannot definitively prove a causal link. The NSQIP database is also limited by the lack of information on pancreatic stents, octreotide, and fibrin glue use. Finally, the DmPJ group was significantly larger $(n=734)$ compared with the IPJ group $(n=156)$, which suggests a national bias and perhaps more experience with this technique.

As for the generalizability of this research, an advantage is that it is the largest study evaluating the two most common methods of pancreatic reconstruction after PD. In the PDP, only $4 \%$ of patients had a PG. In addition, a recent multi-institution, prospective randomized trial from Germany suggests that PG is associated with in- creased postoperative bleeding compared with PJ. ${ }^{36}$ The diversity of the data obtained through the ACSNSQIP PDP may make this study applicable to hepatopancreatobiliary surgeons both in training and in practice. Given the significant mortality differences between DmPJ and IPJ, future investigation into the safest method for reconstruction should be powered to address difference in mortality as well as POPF.

\section{Conclusion}

This analysis suggests that patients undergoing a PJ by DmPJ or IPJ differ with respect to several preoperative and intraoperative variables. Nevertheless, pancreatic fistula rates did not differ significantly, but mortality was significantly greater with an IPJ, particularly among those experiencing pancreatic fistula. Thus, when an invaginated PJ leaks, there may be a significant impact on mortality.

\section{Acknowledgments}

The authors thank surgical reviewers, surgeon champions, and pancreatic surgeons who participated in the Pancreatectomy Demonstration Project at the institutions listed below. They also thank leadership of the American College of Surgeons and of American College of Surgeons-National Surgical Quality Improvement Program for the opportunity to conduct the demonstration project.

$\begin{array}{lcc}\text { Albany Medical Center Kaiser Permanente SF University of Alabama } \\ \text { Baptist Memphis } & \text { Kaiser Walnut } & \text { UC Irvine } \\ \text { Baylor University } & \text { Leigh Valley } & \text { UC San Diego } \\ \text { Baystate Medical } & \text { Massachusetts } & \text { University } \\ \text { Center } & \text { General } & \text { of lowa } \\ \text { Beth Israel Deaconess } & \text { Mayo-Methodist } & \text { University of Kentucky } \\ \text { Boston Medical Center Mayo-St Mary's } & \text { University of Minnesota } \\ \text { Brigham and } & \text { Northwestern } & \text { University Texas } \\ \text { Women's } & \text { University } & \text { Medical Branch } \\ \text { California Pacific } & \text { Ohio State } & \text { University } \\ \text { Medical Center } & \text { University } & \text { of Virginia } \\ \text { Cleveland } & \text { Oregon Health } & \text { University } \\ \text { Clinic } & \text { Sciences Center } & \text { of Wisconsin } \\ \text { Emory } & \text { Penn State } & \text { Vanderbilt } \\ \text { University } & \text { University } & \text { University } \\ \text { Hospital University } & \text { Providence } & \text { Wake Forrest } \\ \text { Pennsylvania } & \text { Portland } & \text { University } \\ \text { Intermountain } & \text { Sacred Heart } & \text { Wash University St. Louis } \\ \text { IU-University } & \text { Stanford University } & \text { Winthrop University } \\ \text { IU-Methodist } & \text { Tampa General } & \\ \text { Johns Hopkins } & \text { Thomas Jefferson } & \\ & \text { University } & \end{array}$

\section{Author Disclosure Statement}

The American College of Surgeons-National Surgical Quality Improvement Program (ACS-NSQIP) and the hospitals participating in the ACS-NSQIP are the 
source of the data used herein; they have not verified and are not responsible for the statistical validity of the data or the conclusions.

\section{References}

1. Raut CP, Tseng JF, Sun CC, et al. Impact of resection status on pattern of failure and survival after pancreaticoduodenectomy for pancreatic adenocarcinoma. Ann Surg. 2007;246:52-60.

2. Siegel R, Naishadham D, Jemal A. Cancer statistics, 2013. CA Cancer J Clin. 2013;63:11-30.

3. Fernández-Cruz L, Belli $A, A$ costa $M$, et al. Which is the best technique for pancreaticoenteric reconstruction after pancreaticoduodenectomy? A critical analysis. Surg Today. 2011;41:761-766.

4. van Berge Henegouwen MI, De Wit LT, Van Gulik TM, et al. Incidence, risk factors, and treatment of pancreatic leakage after pancreaticoduodenectomy: drainage versus resection of the pancreatic remnant. J Am Coll Surg. 1997;185:18-24.

5. Lavu H, Lengel HB, Sell NM, et al. A prospective, randomized, doubleblind, placebo controlled trial on the efficacy of ethanol celiac plexus neurolysis in patients with operable pancreatic and periampullary adenocarcinoma. J Am Coll Surg. 2015;220:497-508.

6. Berger AC, Howard TJ, Kennedy EP, et al. Does type of pancreaticojejunostomy after pancreaticoduodenectomy decrease rate of pancreatic fistula? A randomized, prospective, dual-institution trial. J Am Coll Surg. 2009;208:738-747; discussion 747-749.

7. Bassi C, Butturini G, Molinari E, et al. Pancreatic fistula rate after pancreatic resection. The importance of definitions. Dig Surg. 2004;21:54-59.

8. Fu SJ, Shen SL, Li SQ, et al. Risk factors and outcomes of postoperative pancreatic fistula after pancreatico-duodenectomy: an audit of 532 consecutive cases. BMC Surg. 2015;15:34.

9. Trede M, Schwall G. The complications of pancreatectomy. Ann Surg. 1988;207:39-47.

10. Suzuki Y, Fujino Y, Tanioka $Y$, et al. Selection of pancreaticojejunostomy techniques according to pancreatic texture and duct size. Arch Surg. 2002;137:1044-1047; discussion 1048.

11. Hesse UJ, DeDecker $C$, Houtmeyers $P$, et al. Prospectively randomized trial using perioperative low-dose octreotide to prevent organ-related and general complications after pancreatic surgery and pancreaticojejunostomy. World J Surg. 2005;29:1325-1328.

12. Yang SH, Dou KF, Sharma N, et al. The methods of reconstruction of pancreatic digestive continuity after pancreaticoduodenectomy: a metaanalysis of randomized controlled trials. World J Surg. 2011;35:2290-2297.

13. Kawai $\mathrm{M}$, Yamaue $\mathrm{H}$. Analysis of clinical trials evaluating complications after pancreaticoduodenectomy: a new era of pancreatic surgery. Surg Today. 2010;40:1011-1017.

14. Orci LA, Oldani G, Berney $T$, et al. Systematic review and meta-analysis of fibrin sealants for patients undergoing pancreatic resection. HPB (Oxford). 2014;16:3-11.

15. Chen YJ, Lai EC, Lau WY, et al. Enteric reconstruction of pancreatic stump following pancreaticoduodenectomy: a review of the literature. Int J Surg. 2014;12:706-711.

16. Kennedy EP, Yeo CJ. Dunking pancreaticojejunostomy versus duct-tomucosa anastomosis. J Hepatobiliary Pancreat Sci. 2011;18:769-774.

17. Zollinger RM, Ellison EC. Plate 129. In: Zollinger's Atlas of Surgical Operations, 9e. New York, NY: The McGraw-Hill Companies; 2011. Available at http:// mhmedical.com/content.aspx?aid=6822868 Accessed June 9, 2015.

18. Kim JH, Yoo BM, Kim JH, et al. Which method should we select for pancreatic anastomosis after pancreaticoduodenectomy? World J Surg. 2009; 33:326-332.

19. Zhang JL, Xiao ZY, Lai DM, et al. Comparison of duct-to-mucosa and endto-side pancreaticojejunostomy reconstruction following pancreaticoduodenectomy. Hepatogastroenterology. 2013;60:176-179.

20. Bassi C, Falconi M, Molinari E, et al. Duct-to-mucosa versus end-to-side pancreaticojejunostomy reconstruction after pancreaticoduodenectomy: results of a prospective randomized trial. Surgery. 2003;134:766771.

21. Shiloach M, Frencher SK, Jr, Steeger JE, et al. Toward robust information: data quality and inter-rater reliability in the American College of Surgeons National Surgical Quality Improvement Program. J Am Coll Surg. 2010;210:6-16.
22. Parmar AD, Sheffield KM, Vargas GM, et al. Factors associated with delayed gastric emptying after pancreaticoduodenectomy. HPB. 2013;15:763-772.

23. Lee CW, Pitt HA, Riall TS, et al. Low drain fluid amylase predicts absence of pancreatic fistula following pancreatectomy. J Gastrointest Surg. 2014;18: 1902-1910.

24. Cooper AB, Parmar AD, Riall TS, et al. Does the use of neoadjuvant therapy for pancreatic adenocarcinoma increase postoperative morbidity and mortality rates? J Gastrointest Surg. 2015;19:80-86; discussion 86-87.

25. Beane JD, House MG, Pitt SC, et al. Distal pancreatectomy with celiac axis resection: what are the added risks? HPB. 2015;17:777-784.

26. Tamirisa NP, Parmar AD, Vargas GM, et al. Relative contributions of complications and failure to rescue on mortality in older patients undergoing pancreatectomy. Ann Surg. 2016;263:385-391.

27. Chen JY, Feng J, Wang XQ, et al. Risk scoring system and predictor for clinically relevant pancreatic fistula after pancreaticoduodenectomy. World J Gastroenterol. 2015;21:5926-5933.

28. El Nakeeb A, El Hemaly M, Askr W, et al. Comparative study between duct to mucosa and invagination pancreaticojejunostomy after pancreaticoduodenectomy: a prospective randomized study. Int J Surg. 2015;16:1-6.

29. ACS NSQIP DATA USER GUIDE. Available at www.facs.org/ /media/files/ quality\%20programs/nsqip/ug10.ashx June 2015.

30. Bai XL, Zhang Q, Masood N, et al. Duct-to-mucosa versus invagination pancreaticojejunostomy after pancreaticoduodenectomy: a metaanalysis. Chin Med J (Engl). 2013;126:4340-4347.

31. Noda $\mathrm{H}$, Kamiyama $\mathrm{H}$, Kato $\mathrm{T}$, et al. Risk factor for pancreatic fistula after pancreaticoduodenectomy performed by a surgeon during a learning curve: analysis of a single surgeon's experiences of 100 consecutive patients. Hepatogastroenterology. 2012;59:1990-1993.

32. Fisher WE, Hodges SE, Wu MF, et al. Assessment of the learning curve for pancreaticoduodenectomy. Am J Surg. 2012;203:684-690.

33. El Nakeeb A, Salah T, Sultan A, et al. Pancreatic anastomotic leakage after pancreaticoduodenectomy. Risk factors, clinical predictors, and management (single center experience). World J Surg. 2013;37:1405-1418.

34. Hosotani R, Doi R, Imamura M. Duct-to-mucosa pancreaticojejunostomy reduces the risk of pancreatic leakage after pancreatoduodenectomy. World J Surg. 2002;26:99-104.

35. Zhu B, Geng L, Ma YG, et al. Combined invagination and duct-to-mucosa techniques with modifications: a new method of pancreaticojejunal anastomosis. Hepatobiliary Pancreat Dis Int. 2011;10:422-427.

36. Keck T, Wellner UF, Bahra M, et al. Pancreatogastrostomy versus pancreatojejunostomy for RECOnstruction after PANCreatoduodenectomy (RECO PANC, DRKS 00000767): perioperative and long-term results of a multicenter randomized control trial. Ann Surg. 2016;263:440-449.

Cite this article as: Lavu H, McCall N, Keith SW, Kilbane EM, Parmar A, Hall BL, Pitt HA (2018) Leakage of an invagination pancreaticojejunostomy may have an influence on mortality, Journal of Pancreatic Cancer 4:1, 45-51, DOI: 10.1089/pancan.2018.0008.

Abbreviations Used
ACS-NSQIP $=$ American College of Surgeons-National
Surgical Quality Improvement Program
$\mathrm{BMI}=$ body mass index
$\mathrm{CI}=$ confidence interval
$\mathrm{DGE}=$ delayed gastric emptying
$\mathrm{DJ} / \mathrm{GJ}=$ duodenojejunostomy/gastrojejunostomy
$\mathrm{DmPJ}=$ duct-to-mucosa pancreaticojejunostomy
$\mathrm{DVT}=$ deep vein thrombosis
$\mathrm{IPJ}=$ invagination pancreaticojejunostomy
$\mathrm{OR}=$ odds ratio
$\mathrm{PD}=$ pancreaticoduodenectomy
$\mathrm{PDA}=$ pancreatic ductal adenocarcinoma
$\mathrm{PDP}=$ Pancreatectomy Demonstration Project
$\mathrm{PJ}=$ pancreaticojejunostomy
$\mathrm{POPF}=$ postoperative pancreatic fistula
$\mathrm{SCRS}=$ surgical clinical reviewers
$\mathrm{SSI}=$ surgical-site infection

